\title{
Levantamento epidemiológico dos casos notificados de acidentes por animais peçonhentos no município de Porto Nacional/TO no período de 2013-2018
}

Os acidentes por animais peçonhentos se destacam por ser um dos agravos mais notificados no Brasil, assim como, na cidade de Porto Nacional/To, tornando-se uma emergência clínica comum. Na cidade de Porto Nacional foi registrado no período de 2013 a 2018 um total de 1417 acidentes por animais peçonhentos, resultando em uma média de 236,16 casos por ano. Durante o ano de 2013, foram registrados 166 casos de envenenamentos por animais peçonhentos. Já, em 2018, percebeu-se um crescente aumento nos casos notificados, totalizando 323 casos até o mês de outubro do mesmo ano, destes, a maioria, causados por escorpiões seguidos por serpentes, abelhas, lagarta e aranha. O tratamento é baseado com o auxílio do diagnóstico clínico e identificação do animal causador e os sintomas apresentados pela vítima, como local, extensão, edema, sangramento, manifestação neurotóxica e sistêmica. Visando assim um importante reconhecimento de casos notificados para a saúde pública.

Palavras-chave: Animais Venenosos; Mordeduras de Serpentes; Monitoramento Epidemiológico.

\section{Epidemiological survey of notified cases of accidents by special animals in the municipality of Porto Nacional/TO the period of 2013- 2018}

\begin{abstract}
Accidents caused by venomous animals are notable for being one of the most reported injuries in Brazil, as well as in the city of Porto Nacional/TO, becoming a common clinical emergency. In the city of Porto Nacional, a total of 1417 accidents with venomous animals were recorded in the period from 2013 to 2018 , resulting in an average of 236.16 cases per year. During 2013, 166 cases of venomous animal poisoning were recorded. Already, in 2018, there was a growing increase in reported cases, totaling 323 cases by October of the same year, of which, the majority; caused by scorpions followed by snakes, bees, caterpillar and spider. Treatment is based on the clinical diagnosis and identification of the causative animal and the symptoms presented by the victim, such as location, extent, edema, bleeding, neurotoxic and systemic manifestation. Thus aiming an important recognition of cases notified to public health.
\end{abstract}

Keywords: Poisonous animals; Snake bites; Epidemiological monitoring.

Topic: Epidemiologia

Reviewed anonymously in the process of blind peer
Received: 18/02/2020

Approved: 05/05/2020
Ewerton Charles Barros Dias ii

Faculdade em Porto Nacional, Brasil

http://lattes.cnpq.br/5488657916489963

ewertoncharles@hotmail.com

Vinicius Silveira Dourado (iD

Faculdade Presidente Antônio Carlos, Brasil

http://lattes.cnpq.br/9421795753713568

viniciusdourado@yahoo.com.br
Referencing this:

DIAS, E. C. B.; DOURADO, V. S.. Levantamento epidemiológico dos casos notificados de acidentes por animais peçonhentos no município de Porto Nacional/TO no período de 2013-2018. Scire Salutis, v.10, n.2, p.50-57, 2020. DOI: http://doi.org/10.6008/CBPC2236$\underline{9600.2020 .002 .0007}$ 
Levantamento epidemiológico dos casos notificados de acidentes por animais peçonhentos no município de Porto Nacional/TO no período de 2013-2018

\section{INTRODUÇÃO}

O Brasil é um país continental de grandes diversidades em seu bioma, entre eles, destacam-se os animais peçonhentos os quais são considerados de suma importância em sua abordagem epidemiológica, não sendo diferente esta realidade na cidade de Porto Nacional/TO. São conhecidos por animais peçonhentos, os animais que possuem peçonha, ou seja, têm a capacidade de produzir e expelir toxicidade através de um órgão excretor, inoculando na vítima de sua picada, mordedura ou ferroada uma quantidade significativa de veneno, que varia de espécie para espécie. Os acidentes com escorpiões, serpentes, aranhas, abelhas, besouros, lacraias, águas-vivas e caravelas, estão entre os mais frequentes no Brasil, e possuem sua importância médica em virtude de sua grande frequência e gravidade.

Considerado uma emergência clinica comum, principalmente nos campos e em áreas rurais os acidentes por animais peçonhentos obtiveram destaque na cidade de Porto Nacional/TO, onde se registrou no período de 2013 a 2018 um total de 1417 acidentes por animais peçonhentos, resultando em uma média de 236,16 casos por ano, durante o ano de 2013, foram registrados 166 casos de envenenamentos por esses animais. Já em 2018, percebeu-se um aumento crescente nos casos notificados, num total de 323 casos até o mês de outubro do mesmo ano, destes, a maioria causados por escorpiões, seguidos por serpentes abelhas, lagarta e aranha. Os envenenamentos por animais peçonhentos e suas consequências constituem um problema de Saúde Pública, inclusive pediátrico.

Os escorpiões de interesse médico no estado do Tocantins, cidade de Porto Nacional, constituem-se como gênero Tityus que é a espécie responsável pelo maior número de acidentes leves, moderados e graves no homem e em crianças, até mesmo fatais, são conhecidos como escorpiões marrom (T. bahiensis), escorpiões amarelos (T. serrulatus) e $T$. stigmurus. Os acidentes escorpiônicos apresentam uma diferença significativa entre homens e mulheres, acometendo mais o sexo masculino, ainda não se sabe se por maior exposição devido a trabalhos braçais em zona rural ou apenas fatalidade, sendo as crianças com menos de 10 anos o grupo mais vulnerável (BRASIL, 2006).

As serpentes peçonhentas versadas no Tocantins, cidade de Porto Nacional, e os envenenamentos são classificados em quatro grupos: Botrópico (Gêneros Bothrops e Bothrocophias - jararacas; responsáveis por 116 casos); Crotálico (Gênero Crotalus - cascavéis; responsáveis por 11 casos); Elapídico (Gênero Micrurus - corais-verdadeiras; responsável por apenas 1 dos casos), e Não peçonhentas, responsáveis por 12 casos registrados, baseados na base de dados SINAN no período de 2013 a 2018.

As aranhas conhecidas de importância epidemiológica na região de Porto Nacional/TO, são classificadas em dois gêneros loxoscelismo e latrodectismo, e as aranhas loxoceles (aranha marrom) as que apresentam o veneno mais letal e a que representa maior índice de incidentes dentre as aranhas na área estudada, representando 12 casos notificados no período compreendido entre 2013 a 2018, enquanto as aranhas latrodecttus (flamenguinha representa apenas 1 caso notificado. Os homens são os mais acometidos).

As lagartas que mais acometeram no campo de estudo (cidade de Porto Nacional/TO) é a Lonomia 
onde se registrou um total de 7 casos no período compreendido entre 2013 a 2018, e ainda notificados outros 36 casos de acidentes por lagarta não identificadas. 0 diagnóstico é realizado através da identificação do animal causador do acidente, e dos sintomas apresentados pela vítima, como local, extensão, edema, sangramento, manifestação neurotóxica e sistêmica. Em alguns casos, há recomendação de exame complementar. $\mathrm{O}$ tratamento é sintomático e com soro antiveneno, de acordo com cada espécie (FUNASA, 2001).

A partir das análises dos dados do SINAN, a vigilância epidemiológica é capaz de identificar o quantitativo de soros a serem distribuídos às Unidades Federadas, além de determinar pontos estratégicos de vigilância, estruturar as unidades de atendimento aos acidentados, elaborar estratégias de controle desses animais, entre outros (BRASIL, 2018a).

$\mathrm{Na}$ presente pesquisa, o objetivo é estudar os casos notificados de acidentes por animais peçonhentos no município de Porto Nacional/TO, no período compreendido entre 2013 a 2018, descrevendo o perfil epidemiológico dos casos acometidos por AAP (Acidente por Animais Peçonhentos), expressando a frequência por ano, bairro mais acometido, sexo, classificação de caso, tipo de acidente, local da picada, soroterapia e evolução.

\section{METODOLOGIA}

Estudo descritivo e com exploração quantitativa, transversal que se avaliou epidemiologicamente a frequência de casos registrados de acidentes por animais peçonhentos, cidade de Porto Nacional/TO, e histórico formado pelos anos de '2013 a 2018'. Para elaboração do presente estudo, foi solicitado perante a Secretaria de Vigilância em Saúde (SVS) de Porto Nacional/TO, dados extraídos do Ministério da Saúde (MS), através de informações das fichas do Sistema Nacional de Agravos de Notificação (SINAN). Além disso, buscou-se agregar informações junto à base de dados eletrônicos informatizados, como: Biblioteca Virtual em Saúde (BVS), Literatura Latino-Americana em Ciências da Saúde (LILACS) e Scientific Electronic Library Online (SciELO). Através dos Softwares Microsoft Word e Excel 2010 ${ }^{\circledR}$, pertencente ao pacote Microsoft Office $2010^{\circledR}$, Windows ${ }^{\circledR}$. Os dados levantados foram organizados e expostos por meio de quadros e tabelas explicativas.

\section{RESULTADOS}

Foi identificado um universo de 1.417 casos de acidentes por animais peçonhentos na cidade de Porto Nacional/TO no período analisado de 2013, 2014, 2015, 2016, 2017 e 2018, expostos por bairros, juntamente à tabela de número 1.

Tabela 1: Frequência por ano de notificação segundo bairro residencial 2013-2018.

\begin{tabular}{|l|l|l|l|l|l|l|l|}
\hline BAIRRO RESIDENCIAL & $\mathbf{2 0 1 3}$ & $\mathbf{2 0 1 4}$ & $\mathbf{2 0 1 5}$ & $\mathbf{2 0 1 6}$ & $\mathbf{2 0 1 7}$ & $\mathbf{2 0 1 8}$ & TOTAL \\
\hline TOTAL & 166 & 205 & 210 & 178 & 335 & 323 & 1417 \\
\hline CENTRO & 1 & 8 & 9 & 8 & 17 & 9 & 52 \\
\hline AEROPORTO & - & 5 & 5 & 3 & 6 & 9 & 28 \\
\hline NACIONAL & - & 4 & 4 & - & 4 & 3 & 15 \\
\hline
\end{tabular}


Levantamento epidemiológico dos casos notificados de acidentes por animais peçonhentos no município de Porto Nacional/TO no período de 2013-2018

DIAS, E. C. B.; DOURADO, V.S.

\begin{tabular}{|c|c|c|c|c|c|c|c|}
\hline SÃO JOSE & - & - & - & - & - & 3 & 3 \\
\hline PADRE LUSO & - & 1 & 3 & 2 & 5 & 8 & 19 \\
\hline PARQUE DO TREVO & - & - & - & - & - & 3 & 3 \\
\hline SÃO FRANCISCO & - & 1 & 2 & 3 & 4 & 6 & 16 \\
\hline CONJ. HAB FAMA & - & - & - & - & 2 & 1 & 3 \\
\hline BRIG. EDUARDO GOMES & - & 1 & - & 5 & 3 & 3 & 12 \\
\hline BEIRA RIO & 2 & 2 & - & - & 2 & 3 & 9 \\
\hline ALTO DA COLINA & 1 & 8 & 2 & 5 & 7 & 7 & 30 \\
\hline NOVO HORIZONTE & - & 1 & 1 & 1 & 5 & 2 & 10 \\
\hline NOVO PLANALTO & 1 & 5 & 15 & 2 & 10 & 14 & 47 \\
\hline JARDIM MUNICIPAL & - & 3 & 1 & - & 7 & 4 & 15 \\
\hline SÃO VICENTE & 1 & 2 & 2 & 1 & 5 & 5 & 16 \\
\hline GARCIA & - & 2 & - & - & - & 1 & 3 \\
\hline IMPERIAL & - & 4 & 14 & 10 & 12 & 13 & 53 \\
\hline JARDIM BRASILIA & 4 & 4 & 11 & 4 & 8 & 6 & 37 \\
\hline JARDIM QUERIDO & 3 & 1 & 6 & 7 & 12 & 9 & 38 \\
\hline PARQUE ELDORADO & 3 & 2 & 4 & 2 & 10 & 3 & 24 \\
\hline VILA NOVA & - & 9 & 7 & 3 & 21 & 16 & 56 \\
\hline JARDIM UMUARAMA & - & - & - & - & 4 & 7 & 11 \\
\hline PORTO IMPERIAL & 1 & 5 & 2 & 7 & 9 & 11 & 35 \\
\hline NOVA CAPITAL & 3 & 7 & 4 & 6 & 17 & 22 & 59 \\
\hline JARDIM DOS YPES & - & 2 & - & 2 & 4 & 5 & 13 \\
\hline PRAÇA DAS MÃES & - & 1 & - & - & - & - & 1 \\
\hline CONSORCIO & - & 1 & - & - & - & 2 & 3 \\
\hline TROPICAL PALMAS & - & 5 & - & 2 & 5 & 7 & 19 \\
\hline VILA OPERARIA & 2 & 3 & 6 & - & 5 & 4 & 20 \\
\hline ZONA RURAL & 3 & 22 & 18 & 26 & 42 & 42 & 153 \\
\hline BAIRRO RESIDENCIAL & 2013 & 2014 & 2015 & 2016 & 2017 & 2018 & TOTAL \\
\hline TOTAL & 166 & 205 & 210 & 178 & 335 & 323 & 1417 \\
\hline PINHEIROPOLIS & 1 & 1 & 5 & - & 2 & 2 & 11 \\
\hline SÃO JUDAS TADEU & 1 & - & - & - & - & - & 1 \\
\hline ESCOLA BRASIL & 1 & - & 1 & 1 & 2 & - & 5 \\
\hline ESTAÇÃO DA LUZ & - & - & - & - & 1 & - & 1 \\
\hline PORTO REAL & - & - & 2 & - & - & - & 2 \\
\hline PARQUE DA LIBERDADE & - & 2 & 1 & 2 & 3 & 11 & 19 \\
\hline JARDIM AMERICA & - & 4 & 2 & 3 & 6 & 7 & 22 \\
\hline JARDIM GUAXUPE & - & - & - & - & - & 2 & 2 \\
\hline IRMÃ EDILA & - & - & - & - & - & 5 & 5 \\
\hline VILLAGEM MORENA & - & - & - & - & - & 1 & 1 \\
\hline GRANVILLE & - & - & - & - & - & 2 & 2 \\
\hline RESIDENCIAL JARDINS & - & - & - & - & - & 1 & 1 \\
\hline JARDIM AEROPORTO & - & - & - & - & - & 1 & 1 \\
\hline PRAIA BELA & - & - & - & - & - & 1 & 1 \\
\hline
\end{tabular}

Fonte: BRASIL (2018b).

Na tabela 2, são representados os dados relatados ao quantitativo de casos relacionados à frequência por ano de notificação, segundo o tipo de acidente.

Tabela 2: Tipo de acidente.

\begin{tabular}{|l|l|l|l|l|l|l|l|}
\hline Tipo de Acidente & $\mathbf{2 0 1 3}$ & $\mathbf{2 0 1 4}$ & $\mathbf{2 0 1 5}$ & $\mathbf{2 0 1 6}$ & $\mathbf{2 0 1 7}$ & $\mathbf{2 0 1 8}$ & Total \\
\hline Total & 166 & 205 & 210 & 178 & 335 & 323 & 1.417 \\
\hline Ign/Branco & - & 1 & - & - & 2 & 3 & 6 \\
\hline Serpente & 21 & 25 & 29 & 33 & 25 & 17 & 150 \\
\hline Aranha & 5 & 6 & 6 & 4 & 9 & 17 & 47 \\
\hline Escorpião & 78 & 84 & 76 & 83 & 119 & 109 & 549 \\
\hline Lagarta & 6 & 8 & 7 & 3 & 6 & 20 & 50 \\
\hline Abelha & 17 & 22 & 17 & 3 & 35 & 49 & 143 \\
\hline Outros & 39 & 59 & 75 & 52 & 139 & 108 & 472 \\
\hline
\end{tabular}

Fonte: BRASIL (2018b).

Em relação à frequência por ano da notificação, por sexo, observa-se que, o número de casos 
Levantamento epidemiológico dos casos notificados de acidentes por animais peçonhentos no município de Porto Nacional/TO no período de 2013-2018

DIAS, E. C. B.; DOURADO, V.S.

acometidos no sexo masculino é superior ao feminino, resultando em 853 notificações para homens e 564 para mulheres, totalizando no período de 2013 a 2018, 1417 casos. No ano de 2017 foi a maior taxa para ambos os sexos com 335 casos, sendo 198 para o sexo masculino e 137 para o sexo feminino.

O local da picada é considerado a porta de entrada do veneno, o primeiro local a apresentar reações adversas de acordo com a imunidade e reações de cada indivíduo acometido. Os pés foram os que alcançaram maior índice, totalizando 508 casos com uma média de $38,85 \%$ no período compreendido entre 2013 a 2018, seguidos pelas mãos com 195 casos uma média de 13,76\%, cabeça 180 casos média de 12,70 acidentes, dedos das mãos 128 casos, média de 9,03. E o local menos atingido segundo os dados obtidos no SINAN foi o antebraço com apenas 24 casos e uma média de 1,69\% no mesmo período, os dados encontramse representados na tabela 3.

Tabela 3: Frequência anual de notificação de acordo com o local da picada.

\begin{tabular}{|l|l|l|l|l|l|l|l|}
\hline Local picada & $\mathbf{2 0 1 3}$ & $\mathbf{2 0 1 4}$ & $\mathbf{2 0 1 5}$ & $\mathbf{2 0 1 6}$ & $\mathbf{2 0 1 7}$ & $\mathbf{2 0 1 8}$ & Total \\
\hline TOTAL & 166 & 205 & 210 & 178 & 335 & 323 & 1417 \\
\hline Ign/Branco & 6 & 7 & 9 & 5 & 17 & 10 & 54 \\
\hline Cabeça & 19 & 39 & 21 & 16 & 38 & 47 & 180 \\
\hline Braço & 4 & 8 & 7 & 4 & 7 & 18 & 48 \\
\hline Antebraço & 4 & 3 & 5 & 1 & 6 & 5 & 24 \\
\hline Mão & 27 & 34 & 28 & 21 & 45 & 40 & 195 \\
\hline Dedo Mão & 17 & 18 & 16 & 19 & 28 & 30 & 128 \\
\hline Tronco & 7 & 10 & 12 & 12 & 12 & 29 & 82 \\
\hline Coxa & 3 & 3 & 6 & 4 & 6 & 7 & 29 \\
\hline Perna & 13 & 21 & 19 & 10 & 22 & 22 & 107 \\
\hline Pé & 60 & 53 & 74 & 76 & 141 & 104 & 508 \\
\hline Dedo pé & 6 & 9 & 13 & 10 & 13 & 11 & 62 \\
\hline
\end{tabular}

Fonte: BRASIL (2018b).

Outro dado importante fornecido pelo SINAN, trata-se do grau de gravidade dos casos acometidos pela picada dos animais peçonhentos, classificados em leve, médio e grave, onde constatou-se que, os quadros leves são os mais frequentes correspondendo a $74,17 \%$ dos casos notificados, enquanto o quadro grave apresentou uma média de 1,41\% dos casos revelados que, apesar de alto o número de acidentes por estes animais, os classificados como grave são bem baixos em relação aos quadros leve e médio, representados na tabela 4.

Tabela 4: Classificação dos casos de acordo com o grau de gravidade.

\begin{tabular}{|l|l|l|l|l|l|l|l|}
\hline Classificação do caso & 2013 & 2014 & 2015 & 2016 & 2017 & 2018 & Total \\
\hline Total & 166 & 205 & 210 & 178 & 335 & 323 & 1.417 \\
\hline Ign/ Branco & 2 & - & 1 & 2 & 2 & 4 & 11 \\
\hline Leve & 105 & 156 & 155 & 132 & 248 & 255 & 1.051 \\
\hline Moderado & 57 & 44 & 52 & 41 & 80 & 61 & 335 \\
\hline Grave & 2 & 5 & 2 & 3 & 5 & 3 & 20 \\
\hline
\end{tabular}

Fonte: BRASIL (2018b).

Dependendo do grau de gravidade, o indivíduo acometido pela picada de animais peçonhentos necessita de realização de soroterapia ou não, o que deixa claro e reforça ainda mais a tese de prevalência de casos leves no município de Porto Nacional, onde apenas 293 pessoas receberam a soroterapia o que corresponde a $20,67 \%$ dos casos de soroterapia notificados, enquanto $78,68 \%$ dos casos notificados não receberam a soroterapia. Uma vez que, o tratamento se dá pelo nível de comprometimento, em todos os 
Levantamento epidemiológico dos casos notificados de acidentes por animais peçonhentos no município de Porto Nacional/TO no período de 2013-2018

DIAS, E. C. B.; DOURADO, V.S.

casos é indicado a soroterapia, exceto, nos casos leves escorpiônicos, aracnídeos (foneutrismo, loxocelismo). Portanto, o que caracteriza um quadro leve, são os sinais e sintomas brandos, que geralmente são locais ou até mesmo ausentes, já nos casos moderados e graves, o acometimento em sua maioria são sistêmicos, e de rápida evolução clínica, necessitando assim, maior intervenção médica, e em número significativo de soroterapia conforme protocolo, manual de diagnóstico e tratamento de acidentes por animais peçonhentos. Por fim, pode-se conhecer a evolução dos casos ocorridos no período de 2013 a 2018, evidenciando que, do total de 1417 casos acometidos, 99,64\% evoluíram para cura e apenas 5 casos 0,36\% evoluíram para óbito, sendo que, um dos casos foi a óbito por outra causa, visualizado na tabela 5.

Tabela 5: Evolução dos casos.

\begin{tabular}{|l|l|l|l|l|l|l|l|}
\hline Evolução & $\mathbf{2 0 1 3}$ & $\mathbf{2 0 1 4}$ & $\mathbf{2 0 1 5}$ & $\mathbf{2 0 1 6}$ & $\mathbf{2 0 1 7}$ & $\mathbf{2 0 1 8}$ & Total \\
\hline Cura & 165 & 205 & 209 & 178 & 334 & 321 & 1412 \\
\hline Óbito pelo agravo notificado & 1 & - & 1 & - & 1 & 1 & 4 \\
\hline Óbito por outra causa & - & - & - & - & - & 1 & 1 \\
\hline Total & 166 & 205 & 210 & 178 & 335 & 323 & 1.417 \\
\hline
\end{tabular}

Fonte: BRASIL (2018b).

\section{DISCUSSÃO}

Por meio do contemporâneo estudo, foram identificadas algumas evidências, sendo: (1) o crescimento do quantitativo de registro de acidentes por animais peçonhentos no período exposto, com maior frequência nos anos de 2017 e 2018; (2) a maior incidência em pessoas do sexo masculino; (3) o local de picadura mais acometido foi o pé seguido pela mão; (4) o acidente mais frequente ocorre por escorpiões; (5) a menor incidência de acidentes ocorre com as aranhas; (6) a maioria dos casos são classificados como leves; (7) apenas 20,67\% das pessoas dos 1417 casos necessitaram de soroterapia e (8) a maioria dos casos evoluíram para cura num total 1412 curados.

Proporcionalmente ao aumento do número de casos de acidentes por animais peçonhentos notificados na cidade de Porto Nacional/TO, constata-se o aumento populacional e demográfico associado à urbanização, desmatamento e queimadas, fazendo com que os animais se locomovam para as residências evadindo do local que outrora era seu habitat natural, pode se perceber também que, esse crescente, está diretamente relacionada ao aumento das notificações efetuadas nesse período de estudo, evidenciando uma maior efetividade da vigilância sanitária e melhor acesso das pessoas às unidades de saúde da família.

No estudo em questão, demonstra que foi registrado nos períodos de 2013 a 2018 um total de 1417 acidentes por animais peçonhentos, resultando em uma média de 236,16 casos por ano, desses acidentes, a maioria ocasionados por escorpião, somatizando um total de 549 picadas neste período e uma média de 91,5 casos anuais, notificou-se ainda um total de 472 incidentes com estes animais caracterizado como outros (a pessoa não soube identificar qual o tipo de animal se acidentou). Os acidentes com serpentes resultaram em um total de 150 no mesmo período e uma média anual de 25 notificações, em quarto lugar foi notificado 143 acidentes com abelhas uma média de $23,83 \%$ casos anuais, seguido por 50 ocorrências por acidente com lagarta uma média de 8,33\% ao ano, e por fim, 47 acidentes por picadas de aranha em uma média de 7,83\% incidências ao ano. 
Levantamento epidemiológico dos casos notificados de acidentes por animais peçonhentos no município de Porto Nacional/TO no período de 2013-2018

DIAS, E. C. B.; DOURADO, V.S.

A frequência por ano da notificação segundo o sexo, se expressa em uma incidência de 289 acidentes a mais para o sexo masculino, representados por um total de 853 casos entre os períodos de 2013 a 2018, com uma média de $142,16 \%$ ao ano, enquanto o sexo feminino abrangeu um total de 564 casos, com uma média de 94 casos anuais.

De acordo com os dados obtidos no SINAN, (Sistema de Informação de Agravos de Notificação), o local de picada mais frequente é o pé com 508 casos notificados, seguido pela mão com 195, cabeça com 180, dedo da mão com 128, perna 107, tronco 86, dedo do pé 62 , braço 48, coxa 29 e antebraço com 24 casos, em 54 notificações, o campo de local da picada não foi preenchido.

Durante o período compreendido de estudo os casos leves atingiram um total de 1051 casos, já os acidentes considerados moderados representaram um total de 335 casos enquanto os graves somaram apenas 20 incidências. Destes agravos somente 293 receberam soroterapia e quatro evoluíram para óbito pelo agravo notificado e um evoluiu para óbito por outra causa, os outros 1412 evoluíram para cura.

Fica claro que, a maior parte dos acidentados por animais peçonhentos realizou acompanhamento e tratamento seja nas unidades de saúde da família ou no hospital regional, o que é eficaz, uma vez que o atendimento precoce é um fator protetivo à saúde, entretanto, apesar do notável crescente de casos notificados se percebe que, ainda existem muitos campos do formulário de notificação não preenchidos ou preenchidos de forma equivocada, o que ainda precisa ser trabalhado entre as equipes de saúde da família, hospitais e demais locais onde são preenchidos os formulários de notificação compulsória, visualmente percebendo a necessidade de capacitação a esses profissionais. É importante salientar que, a prevenção é uma das principais maneiras de evitar um acidente por animal peçonhento, os principais cuidados consistem em evitar ambientes sujos ou entulhados, realizando sempre uma boa limpeza destes locais (BRASIL, 2016).

\section{CONCLUSÕES}

Em virtude dos fatos mencionados é possível identificar os variados tipos de animais peçonhentos e a forma pela qual acontece a picada, levando a crer que, este problema não afeta apenas a zona rural, abrangendo também de forma crescente as cidades e principalmente lugares que contem sujeira, entulhos e próximos as matas.

Este estudo foi de suma importância para o conhecimento acerca dos acidentes por animais peçonhentos, assim como o aprendizado através das notificações na cidade de Porto Nacional/TO, pela vigilância epidemiológica e do SINAN, onde foi possível verificar e conhecer a frequência por ano de notificação, e os principais tipos de acidentes, dentre eles, os por serpentes, aranhas, escorpiões, lagartas, abelhas entre outros. A frequência por sexo onde se constatou que o sexo masculino é o mais acometido, a frequência por ano da notificação segundo o local da picada, bem como a frequência de notificação segundo a classificação de risco entre leve, médio e grave, a quantidade de pessoas que utilizaram a soroterapia como tratamento, e a evolução do caso para a cura ou óbito, compreendidos entre o período de estudo de 20132018. 
Pode se constatar a necessidade de capacitação para os profissionais que preenchem as fichas de notificação compulsória, apesar do número crescente de notificações no decorrer dos anos de 2013-2018, as notificações ainda apresentam falhas, como não preenchimentos de dados importantes, acarretando em informações incompletas apresentadas nas tabelas como ignorado, branco ou outros.

Visualiza-se, por meio das informações analisadas, que, ainda, há uma extensa jornada a ser percorrida para se impetrar a meta nacional de controle desse importante agravo à saúde pública, que é reduzir as notificações e diminuir os casos fatais. Para que ocorra uma transformação nesse cenário, é preciso focar na educação e treinamento, junto com à comunidade e profissionais de saúde.

\section{REFERÊNCIAS}

BRASIL. Fundação Nacional de Saúde. Manual de saneamento. 3 ed. Brasília: Fundação Nacional de Saúde, 2006.

BRASIL. Ministério da Saúde. Sistema de Informação de Agravos de Notificação - SINAN: normas e rotinas. Brasília: Ministério da Saúde, 2018a.
BRASIL. Ministério da Saúde. Guia de Vigilância Epidemiológica. Brasília: Ministério da Saúde, 2018b.

FUNASA. Fundação Nacional de Saúde. Manual de diagnóstico e tratamento de acidentes por animais peçonhentos. Brasília: FUNASA, 2001.

A CBPC - Companhia Brasileira de Produção Científica (CNPJ: 11.221.422/0001-03) detém os direitos materiais desta publicação. Os direitos referem-se à publicação do trabalho em qualquer parte do mundo, incluindo os direitos às renovações, expansões e disseminações da contribuição, bem como outros direitos subsidiários. Todos os trabalhos publicados eletronicamente poderão posteriormente ser publicados em coletâneas impressas sob coordenação da Sustenere Publishing, da Companhia Brasileira de Produção Científica e seus parceiros autorizados. Os (as) autores (as) preservam os direitos autorais, mas não têm permissão para a publicação da contribuição em outro meio, impresso ou digital, em português ou em tradução. 